Article

\title{
Guided-Mode-Leaky-Mode-Guided-Mode Fiber Interferometer and Its High Sensitivity Refractive Index Sensing Technology
}

\author{
Qi Wang ${ }^{1,2}{ }^{2}$, Chunyue $\mathrm{Li}^{1}$, Chengwu Zhao ${ }^{1}$ and Weizheng $\mathrm{Li}^{1}$ \\ 1 College of Information Science and Engineering, Northeastern University, Shenyang 110819, China; \\ 20123553@stu.neu.edu.cn (C.L.); 20123645@stu.neu.edu.cn (C.Z.); 20133541@stu.neu.edu.cn (W.L.) \\ 2 State Key Laboratory of Synthetical Automation for Process Industries, Northeastern University, \\ Shenyang 110819, China \\ * Correspondence: wangqi@ise.neu.edu.cn; Tel.: +86-138-8919-5280
}

Academic Editor: Vittorio M. N. Passaro

Received: 8 March 2016; Accepted: 20 May 2016; Published: 1 June 2016

\begin{abstract}
A cascaded symmetrical dual-taper Mach-Zehnder interferometer structure based on guided-mode and leaky-mode interference is proposed in this paper. Firstly, the interference spectrum characteristics of interferometer has been analyzed by the Finite Difference-Beam Propagation Method (FD-BPM). When the diameter of taper waist is $20 \mu \mathrm{m}-30 \mu \mathrm{m}$, dual-taper length is $1 \mathrm{~mm}$ and taper distance is $4 \mathrm{~cm}-6 \mathrm{~cm}$, the spectral contrast is higher, which is suitable for sensing. Secondly, experimental research on refractive index sensitivity is carried out. A refractive index sensitivity of $62.78 \mathrm{~nm} / \mathrm{RIU}$ (refractive index unit) can achieved in the RI range of 1.3333-1.3792 $(0 \% \sim 25 \% \mathrm{NaCl}$ solution), when the sensor structure parameters meet the following conditions: diameter of taper waist is $24 \mu \mathrm{m}$, dual-taper length is $837 \mu \mathrm{m}$ and taper distance is $5.5 \mathrm{~cm}$. The spectrum contrast is 0.8 and measurement resolution is $1.6 \times 10^{-5}$ RIU. The simulation analysis is highly consistent with experimental results. Research shows that the sensor has promising application in low RI fields where high-precision measurement is required due to its high sensitivity and stability.
\end{abstract}

Keywords: optical fiber sensor; refractive index measurement; Micro-Nano fiber; Mach-Zehnder interferometer; high sensitivity

\section{Introduction}

The refractive index (RI) is a significant physical parameter to reflect the characteristics of material [1,2]. RI measurement is also important in many application fields such as the detection of solution concentration in the chemical field, water quality monitoring in the environmental field, and real-time monitoring cell culture medium in the biology field. In recent years, because of the advantages of compact structure, small size, light weight, rapid response, high sensitivity, low loss, anti-electromagnetic interference and applicability for remote sensing, fiber optics sensing technology have been widely studied and are becoming a hot research topic [2-4]. There have been many reports on optical fiber sensors which were used for measuring liquid RI, but the sensitivities of these sensors are not high enough in low RI (near 1.33) liquid or the structures are complex [5]. In 2008, Zhaobing Tian put forward a new type fiber RI sensors based on two positive-cascaded $3 \mathrm{~dB}$ tapers Mach-Zehnder interferometer by optical fiber fusion splicer [6]. The experiment shows that the spectrum contrast is $23.4 \mathrm{~dB}$ and RI sensitivity is $17.1 \mathrm{~nm} / \mathrm{RIU}$ when L is $36 \mathrm{~mm}$. In 2009, Ping Lu made two conical structures in a single mode fiber to build a Mach-Zehnder interferometer [7] for simultaneous measurement of RI and temperature, and the RI sensitivity can reach $26.087 \mathrm{~nm} / \mathrm{RIU}$. In 2011, Rao introduced a piece of slow-taper and weak-pulling taper area into the middle of two cascaded tapers to 
form a "sandwich" structure [8]. The principle is that the middle structure can enhance the evanescent field of optical energy and increase the exposed energy of the light field for achieving the improvement of RI sensing sensitivity, and a RI sensitivity of $28.6 \mathrm{~nm} / \mathrm{RIU}$ has been realized in the RI range of 1.3320-1.3890.

Although these sensors show high sensitivities in the high RI measurements field (near the RI of silica), it can hardly meet the high-precision measurement requirement in low RI measurement field. In this paper, we proposed a Mach-Zehnder interferometer structure based on the interference between modes. When the diameter of the taper waist is $24 \mu \mathrm{m}$, dual-taper length is $837 \mu \mathrm{m}$ and taper distance is $5.5 \mathrm{~cm}$, the RI sensitivity is $62.78 \mathrm{~nm} / \mathrm{RIU}$ in the RI range of $1.3333-1.3792$. The spectrum contrast is 0.8 and measurement resolution is $3 \times 10^{-4}$ RIU. The results show that simulation results and experimental are highly consistent. In this paper, the fabrication process of the sensor is simple and fast, the cost is low, and the RI sensitivity of the sensor is higher, which is expected to be widely used in the field of stress [9,10], temperature [11], and so on.

\section{Preparation and Principle}

\subsection{Preparation of Fiber Interferometer}

A simple and feasible, low cost and good repeatability fiber dual-taper making process should be devised as soon as possible [12]. A new method is proposed in this paper, which only needs a fusion splicer (S178, FITEL, FURUKAWA ELECTRIC CO., LTD., Tokyo, Japan) and a high precision micro displacement platform (NFP-x462, ZOLIX, ZOLIXINSTRUMENTS CO.LTD., Beijing, China). The fabrication process of the interferometer is shown in Figure 1.

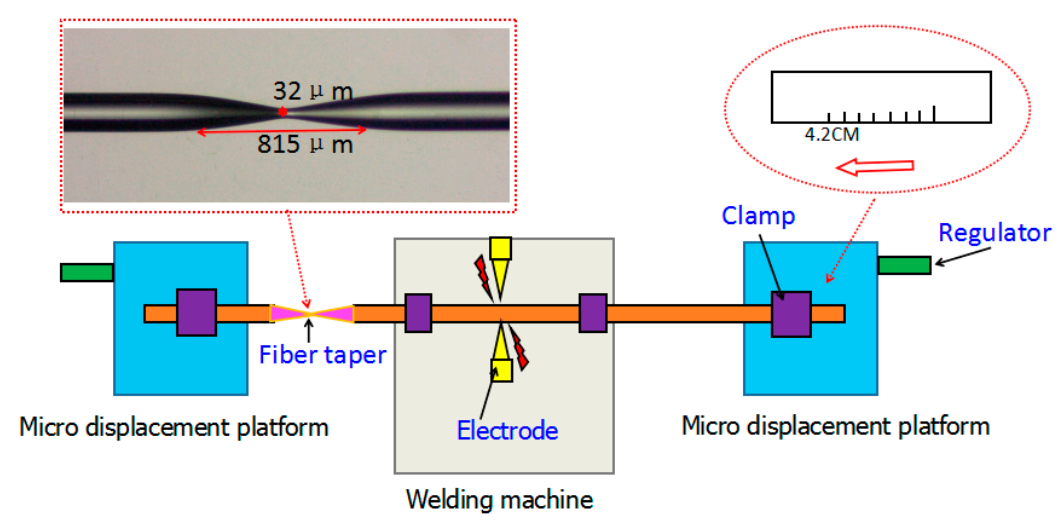

Figure 1. Cascaded symmetrical dual-taper preparation of Mach-Zehnder interferometer.

Firstly, a $5 \mathrm{~cm}$ length coating layer of single-mode fiber was removed by using a miller clamp. Then, we cleaned the surface of the fiber by using lens paper with alcohol and pretreated the fiber without coating layer in high temperature for $5 \mathrm{~min}$ in a fusion splicer. Finally, we used the fusion splicer to control tapered fiber structure parameters such as taper-area length, taper angle and diameter of taper waist. In the first experiment, we got a taper with taper area length of $816 \mu \mathrm{m}$ and taper waist diameter of $32 \mu \mathrm{m}$, which is shown in Figure 1. The transmitted power is $-26.03 \mathrm{dBm}$ when the fiber was integrated, and the total transmitted power is $-29.39 \mathrm{dBm}$ after drawing a dual-taper structure. The interference phenomenon appeared when we made a second taper which is $4.2 \mathrm{~cm}$ away from the first one, and the total power of the interferometer is $-32.23 \mathrm{dBm}$. The spectrum of backward incidence is basically consistent with that of forward incidence, which proved that the performance of the interferometer structure above is stable.

\subsection{Sensing Principle of Fiber Interferometer}

In this paper, the cascaded dual-taper fiber structure forms a Mach-Zehnder interferometer. The fundamental mode in fiber core excites high-order modes in the first taper area and couples into the cladding. Then the high-order modes transmitted in cladding produce a strong evanescent field in 
the interface of cladding and external environment. Fundamental mode and high-order modes with external environment RI information will couple with each other. Due to the different effective RI between cladding modes and core mode, a phase difference will come up after the light transmitting a distance L. So this cascaded dual-taper structure forms a Mach-Zehnder interferometer. Figure 2 shows the tapered fiber Mach-Zehnder interferometer, and the interval length of two tapers (interference length) is $\mathrm{L}$.

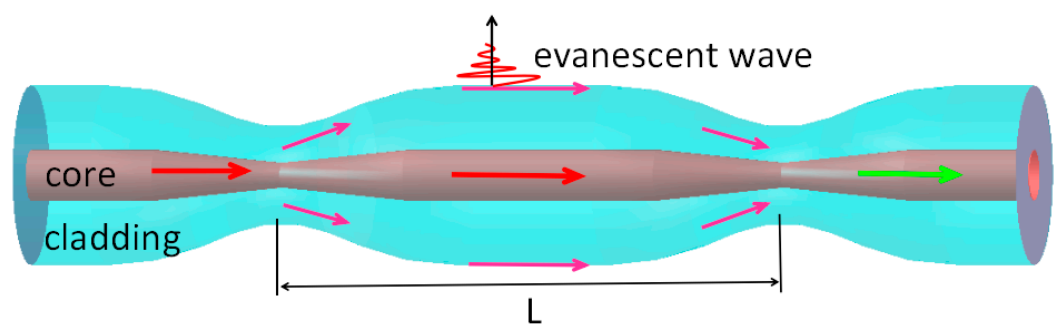

Figure 2. Principle diagram of dual-taper Mach-Zehnder interferometer.

Because many transport modes exist in the cladding and only the fundamental mode exists in the fiber core, usually one of the cladding modes interferences with fundamental mode when analyzing the interference pattern. Therefore, the interference intensity between fundamental mode and cladding mode can be expressed with double-beam interference principle [13,14].

$$
\begin{aligned}
& I=I_{1}+I_{2}+2 \sqrt{I_{1} I_{2}} \cos \left(\varphi+\varphi_{0}\right) \\
& \varphi=\frac{2 \pi\left(n_{\mathrm{co}}-n_{\mathrm{cl}}\right) L}{\lambda}=\frac{2 \pi \Delta n_{\mathrm{eff}} L}{\lambda}
\end{aligned}
$$

Among them, $I_{1}$ and $I_{2}$ are beam intensity of fundamental mode and cladding mode, $\varphi$ is the phase difference between fundamental mode and cladding mode, $\varphi_{0}$ is the initial phase, $n_{\text {co }}$ is the RI of fiber core mode and $n_{\mathrm{cl}}$ is the RI of cladding mode, $\Delta n_{\mathrm{eff}}$ is the difference of RIs, $L$ is the length of interferometer, $\lambda$ is the input wavelength. From Equation (1), we can see that the contrast ratio of interference fringe is depended on the ratio between $I_{1}$ and $I_{2}$.

The contrast ratio of interference fringe can reach maximum value when the phase difference satisfies the condition of $\varphi+\varphi_{0}=(2 m+1) \pi$, and the light intensity of interference reach minimum value. The corresponding interference dip wavelength can be expressed as Equation (3).

$$
\lambda_{m}=\frac{2 \Delta n_{\mathrm{eff}} L}{2 m+1}
$$

The RI of the solution around the fiber will increase when the sensor is immersed in a solution, it will cause the change of cladding mode normalized frequency. Then the mode field distribution and effective RI will change. Because the fundamental mode of the fiber core is almost not affected by the RI change of external environment, the effective RI difference between fiber core and cladding mode will change, which will cause a shift of interference spectrum dip. Thus, we can realize an accurate measurement for external environment RI. The RI measurement sensitivity is associated with Mach-Zehnder interferometer structure. Moreover, the measuring range of Mach-Zehnder interferometer is also an important indicator which is usually measured by the distance between adjacent dips. The greater the distance between dips, the greater the spectrum measurement range will be, and the more difficult it will be to generate mixing. In theory, the free spectrum range $\Delta \lambda_{m}$ can be expressed as Equation (4).

$$
\Delta \lambda_{m}=\lambda_{m}-\lambda_{m-1} \approx \frac{\lambda^{2}}{\Delta n_{\mathrm{eff}} \cdot L}
$$




\section{Simulation Analysis of Fiber Mach-Zehnder Interferometer}

\subsection{Spectrum Characteristic Simulation Analysis of Fiber Mach-Zehnder Interferometer}

We analyzed the cascaded dual-taper interference spectrum and RI sensitivity. The adjustable parameters of the structure mainly include cascaded dual-taper area length $\Delta$, diameter of taper waist $\mathrm{D}$ and distance between two tapers L, as shown in Figure $3 \mathrm{~d}$.

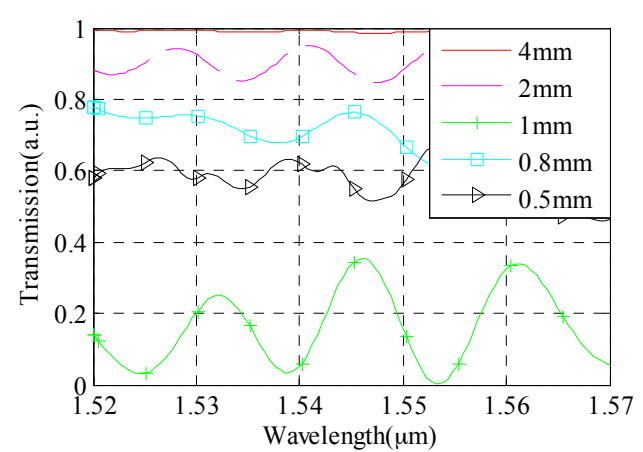

(a)

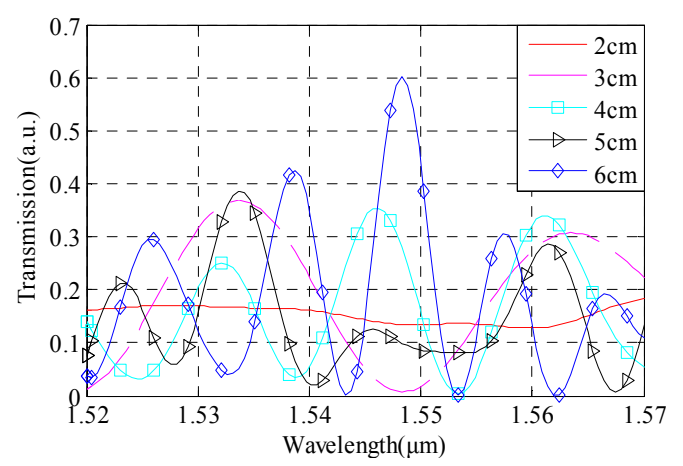

(c)

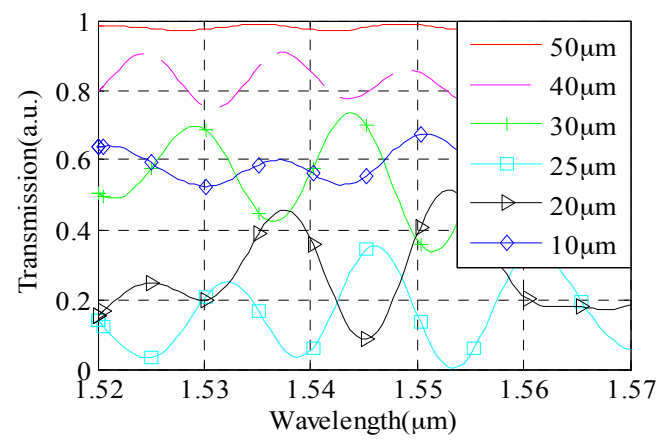

(b)

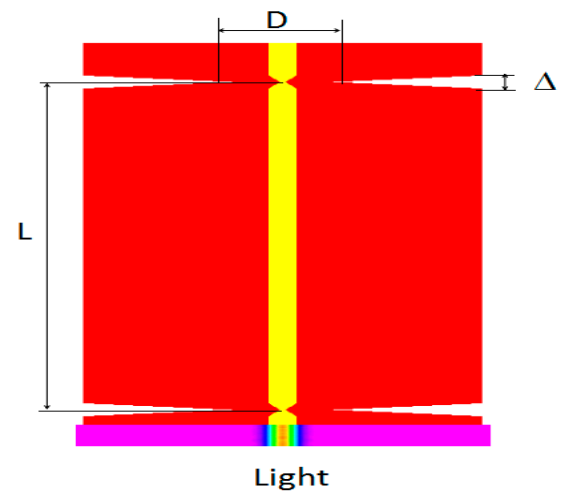

(d)

Figure 3. The simulation model and interference spectrum with different structural parameters. (a) Different taper area length $\Delta$; (b) Different taper waist diameter D; (c) Different cone distance L corresponding; (d) The simulation model.

Figure 3a shows the influence of taper length on spectrum contrast. Firstly, we simulate and analyze the distribution of field excited by tapers, and find that the excitation is better when the diameter of taper is $25 \mu \mathrm{m}$. So, we set the diameter as $25 \mu \mathrm{m}$, and the distance between two tapers is $4 \mathrm{~cm}$. We get the interference spectrum of cascaded dual-taper when the taper length varies among $0.5 \mathrm{~mm}, 0.8 \mathrm{~mm}, 1 \mathrm{~mm}, 2 \mathrm{~mm}$, and $4 \mathrm{~mm}$. As shown in Figure 3a, the transmitted spectrum does not show visible interference when the length of taper is $4 \mathrm{~mm}$. When the taper length decreases to $2 \mathrm{~mm}$, visible interference appears. However, the contrast of the spectrum is low, and we can not find the shift of taper length and dip with the change of RI. Moreover, all of the change cannot be found when the taper length decreases to $500 \mu \mathrm{m}$. Only when the taper length is $1 \mathrm{~mm}$, are the contrast of interference spectrum and free spectrum range better for the study of the change of taper length and dip with RI variation. Because of these, we mainly study RI sensing characteristics of cascaded dual-taper whose length is $1 \mathrm{~mm}$.

Figure $3 \mathrm{~b}$ shows taper diameter influence on spectrum. According to the analysis above, we set the taper length as $1 \mathrm{~mm}$, the distance between two tapers as $4 \mathrm{~cm}$. From Figure $3 \mathrm{~b}$ we can find that when the taper diameter is $50 \mu \mathrm{m}$, the spectrum contrast is too low. When the taper diameter decreases gradually, the spectrum contrast becomes better. In this process, we will get an ideal contrast when the 
taper diameter varies from $30 \mu \mathrm{m}$ to $20 \mu \mathrm{m}$. So we mainly research RI sensing characteristics with the taper diameter varying from $30 \mu \mathrm{m}$ to $20 \mu \mathrm{m}$.

Figure $3 \mathrm{c}$ shows the distance of two tapers influence on spectrum contrast. As in the simulation described above, it will get a better proportion of light coupling when the diameter is $25 \mu \mathrm{m}$. In this paper, we set the length of the taper as $1 \mathrm{~mm}$, diameter of the taper as $25 \mu \mathrm{m}$. We can obtain interference spectrum when $L$ varies among $4 \mathrm{~cm}, 3 \mathrm{~cm}, 5 \mathrm{~cm}, 6 \mathrm{~cm}$ and $2 \mathrm{~cm}$. From Figure $3 \mathrm{c}$ we can see that the taper distance gradually increases from $2 \mathrm{~cm}$ to $6 \mathrm{~cm}$, the interference spectrum is becoming more and more intensive, and the free spectrum range is becoming smaller and smaller, which is consistent with the theoretical analysis results. Although the RI measurement sensitivity of cascade dual-taper structure will increase with the increase of tapers distance, the taper distance can not be too long, because sensor mechanical strength will decrease as the taper distance increases.

According to the simulation analysis, we obtained the better cascade dual-taper structure parameters, and taper waist diameter range is determined as $20 \mu \mathrm{m}-30 \mu \mathrm{m}$ and taper distance range is determined as $4 \mathrm{~cm}-6 \mathrm{~cm}$, then we analyzed the cascade dual-taper interferometer RI sensitivity with different structural parameters.

\subsection{Simulation Analysis on Mach-Zehnder Sensor Refractive Index Sensitivity}

In this experiment, $\mathrm{NaCl}$ solution is more easy to achieve the mass percentage concentration range $0 \%-25 \%$, the corresponding $\mathrm{RI}$ range is $1.3333-1.3792$. In this paper, we set the solution $\mathrm{RI}$ of $1.34,1.35,1.36,1.37,1.38$ respectively. The length of the taper area is $1 \mathrm{~mm}$, diameter of the taper is $30 \mu \mathrm{m}, 25 \mu \mathrm{m}$ and $20 \mu \mathrm{m}$ respectively, and the distance of the tapers is $5 \mathrm{~cm}$.

We obtained the transmission spectrum of different RIs and analyzed the sensitivities of the sensor with different structure parameters. The linear fitting relationships between wavelength of interference valley and RI are shown in Figure $4 \mathrm{a}-\mathrm{c}$, and the corresponding RI measurement sensitivities can reach $50.5 \mathrm{~nm} / \mathrm{RIU}, 76.3 \mathrm{~nm} / \mathrm{RIU}$, and $105.3 \mathrm{~nm} / \mathrm{RIU}$, respectively. Thus, in the diameter range of $20 \mu \mathrm{m}$ to $30 \mu \mathrm{m}$, the smaller taper diameter, the higher sensitivity.

Therefore, we needed to fabricate a series of cascade dual-taper structures. Figure $4 \mathrm{~b}$ is the interference spectrum when taper waist diameter is $25 \mu \mathrm{m}$. There are only three dips in the wavelength range of $1520 \mathrm{~nm}-1570 \mathrm{~nm}$, which proves that the structure parameters of free spectrum range is better.

The length of the taper area is $1 \mathrm{~mm}$, the distance of the tapers is $4 \mathrm{~cm}, 5 \mathrm{~cm}$ and $6 \mathrm{~cm}$ respectively, and the diameter of the taper is $25 \mu \mathrm{m}$. We obtained the transmission spectrum of different RIs and analyzed the sensitivities of the sensor with different structure parameters. The linear fitting relationships between wavelength of interference valley and RI are shown in Figures $4 b$ and $5 a, b$, and the corresponding RI measurement sensitivities can reach $46.1 \mathrm{~nm} / \mathrm{RIU}, 76.3 \mathrm{~nm} / \mathrm{RIU}$, and $87.5 \mathrm{~nm} / \mathrm{RIU}$, respectively. Thus, in the tapers distance of $4 \mathrm{~cm}$ to $6 \mathrm{~cm}$, the longer taper distance has the highest sensitivity.
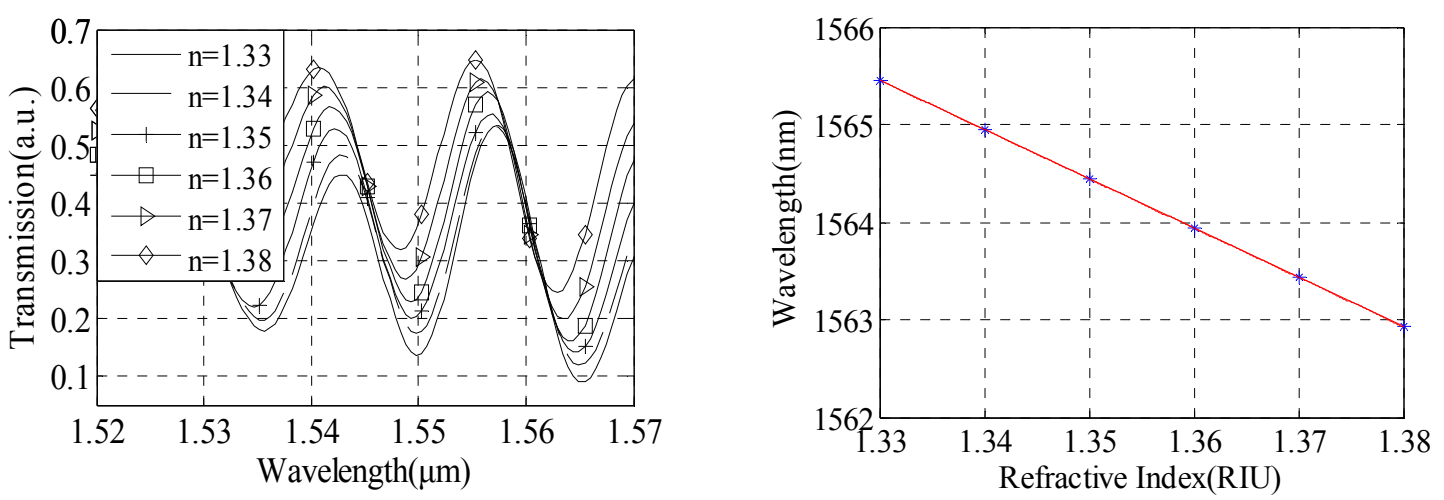

(a)

Figure 4. Cont. 

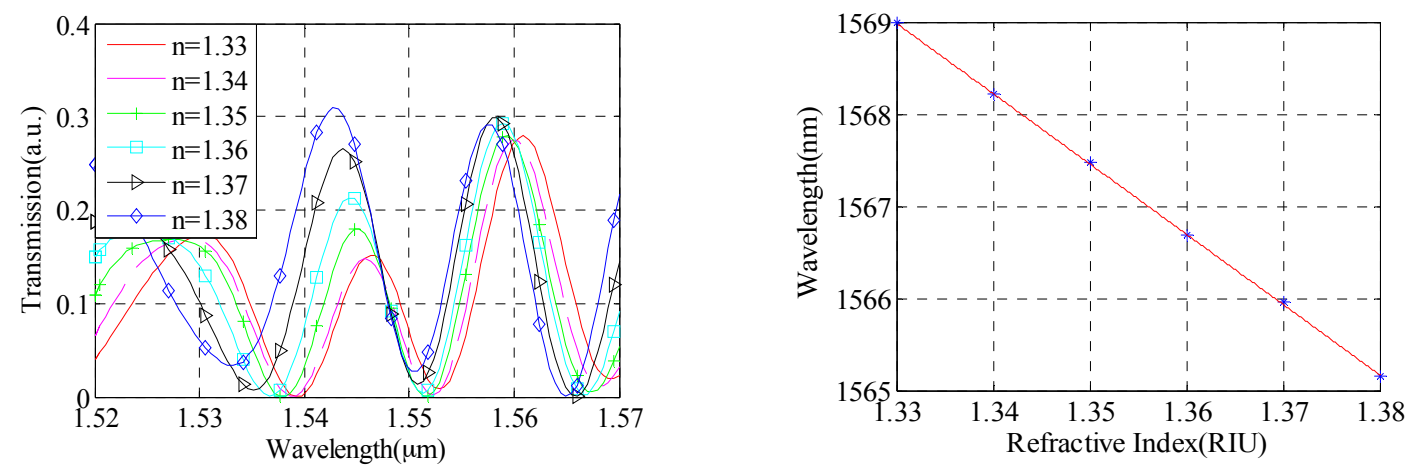

(b)
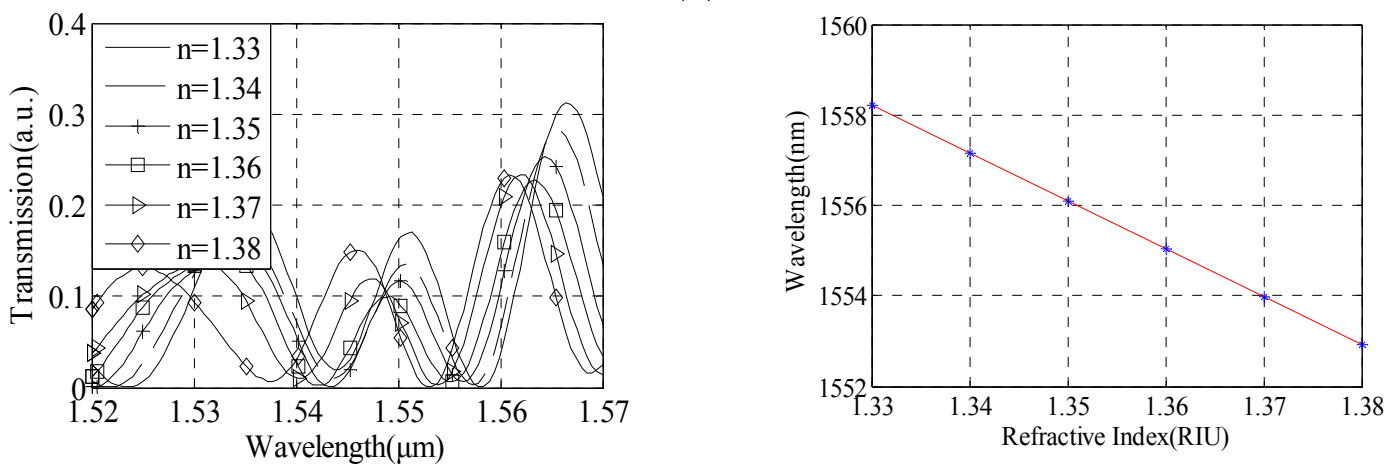

(c)

Figure 4. Refractive index measurement sensitivity analysis with different taper waist diameters. (a) $\mathrm{D}=30 \mu \mathrm{m}$; (b) $\mathrm{D}=25 \mu \mathrm{m}$; (c) $\mathrm{D}=20 \mu \mathrm{m}$.
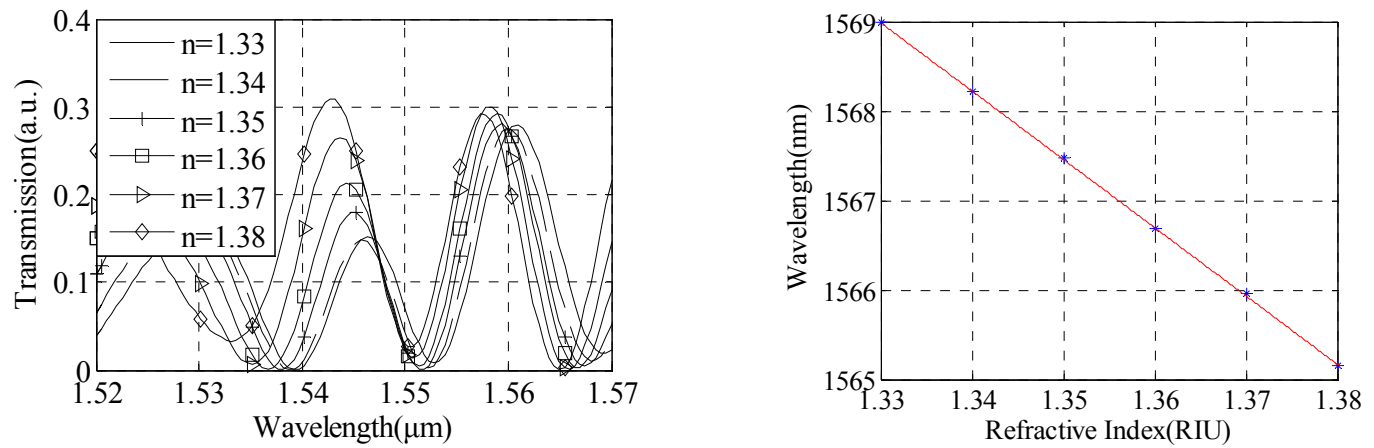

(a)
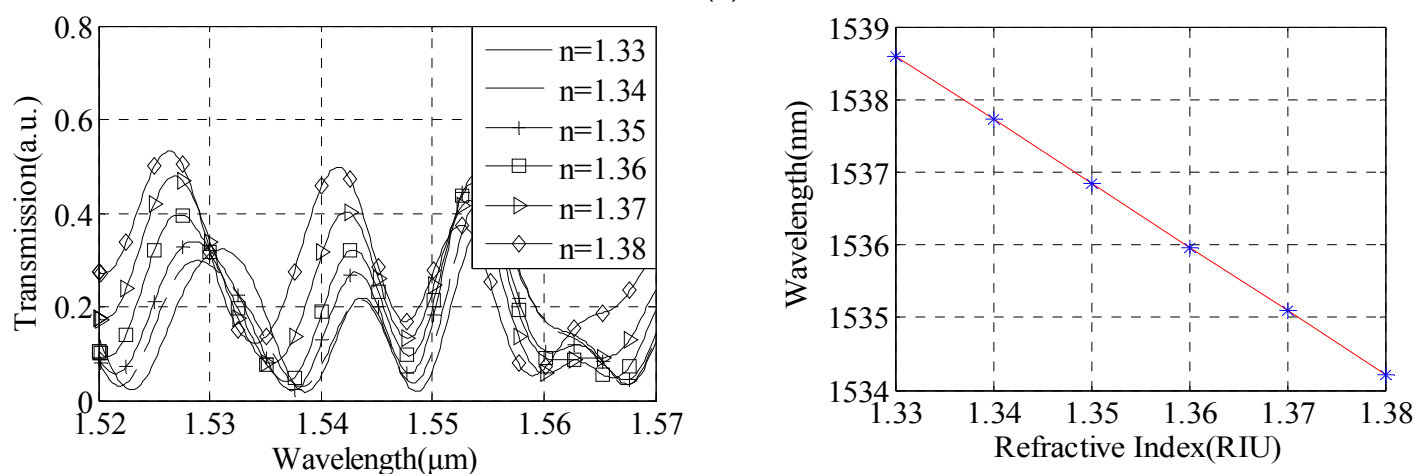

(b)

Figure 5. Refractive index measurement sensitivity analysis with different distance of the tapers. (a) $\mathrm{L}=4 \mathrm{~cm}$; (b) $\mathrm{L}=6 \mathrm{~cm}$. 


\section{Experiment Results Analysis on Refractive Index Measurement}

The experimental device based on cascaded dual-taper Mach-Zehnder interferometer is shown in Figure 6. The experimental system includes an ASE broadband light source with a wavelength range of $1520 \mathrm{~nm}-1570 \mathrm{~nm}$, an optical spectrum analyzer (AQ6370B, YOKOGAWA, Yokogawa Electric Corporation, Tokyo, Japan) and a liquid box with temperature controller which ensures that the experiment is carried out under constant temperature.

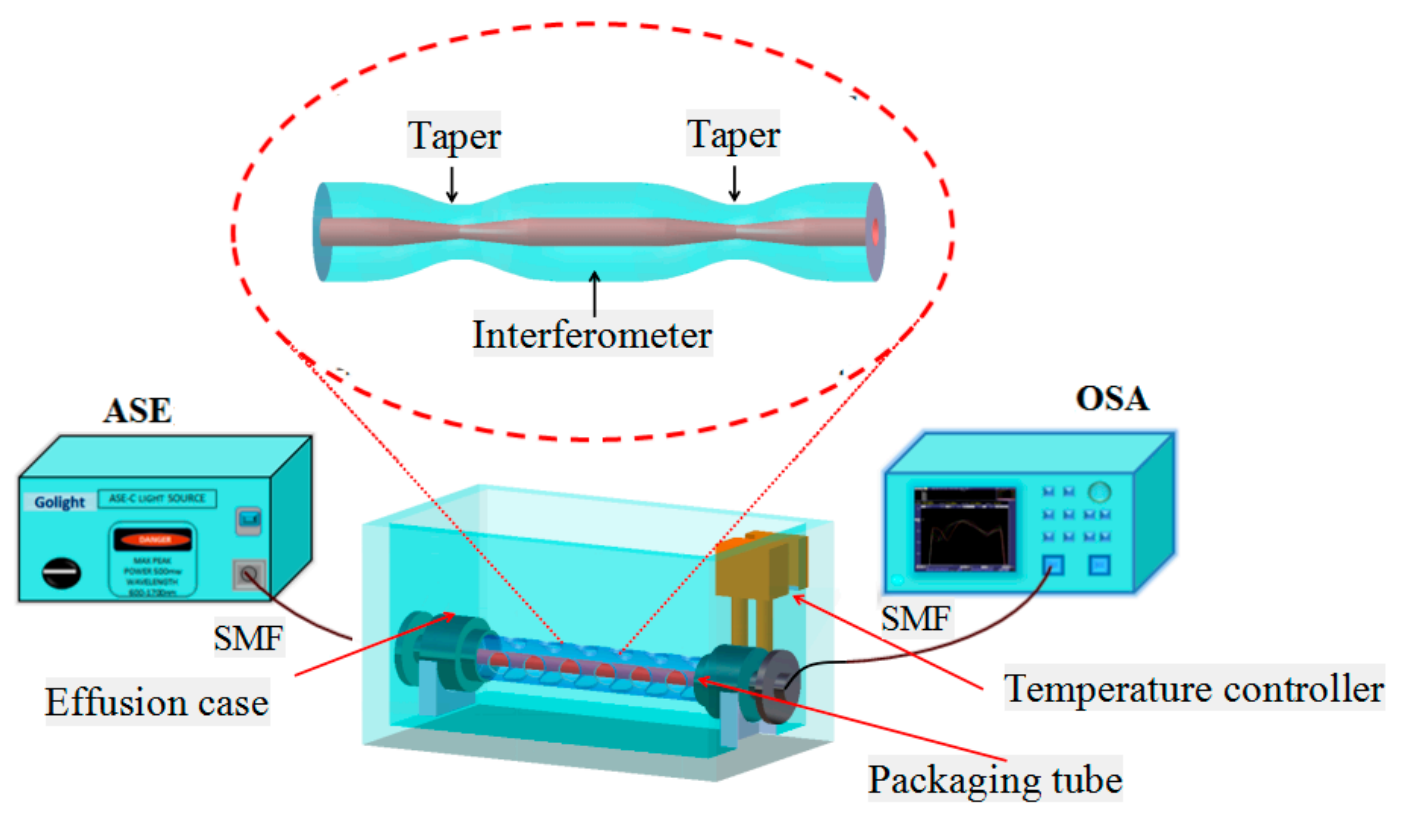

Figure 6. Laboratory equipment schematic of refractive index sensing system.

The $\mathrm{NaCl}$ solution was used as a detected solution, and the corresponding relationship between RI and solution concentration was obtained as shown in Table 1 [15].

Table 1. $\mathrm{NaCl}$ Solution refractive index table along with the change of concentration.

\begin{tabular}{ccccccc}
\hline$c / \%$ & $\mathbf{0}$ & $\mathbf{5}$ & $\mathbf{1 0}$ & $\mathbf{1 5}$ & $\mathbf{2 0}$ & $\mathbf{2 5}$ \\
\hline Refractive Index & 1.3333 & 1.3424 & 1.3510 & 1.3609 & 1.3708 & 1.3792 \\
\hline
\end{tabular}

Based on the theory and simulation analysis, a series of experiments were carried out to study the relationship between RI sensitivity and structure parameters of the sensor. When the diameter of taper waist is less than $20 \mu \mathrm{m}$, the fiber is broken easily, and encapsulation process is complex. Therefore, we make an interferometer with a diameter of $20 \mu \mathrm{m}-30 \mu \mathrm{m}$ in our experiments. The interferometer with a diameter of $25 \mu \mathrm{m}$ had different taper distances of $4 \mathrm{~cm}-6 \mathrm{~cm}$. The results show that the experimental results are consistent with the simulation.

Figure 7 is the sensor sensitivity with taper waist diameter of $20 \mu \mathrm{m}-30 \mu \mathrm{m}$, taper distance of $5 \mathrm{~cm}$, and taper area length of $818 \mu \mathrm{m}$. When taper waist diameter $\mathrm{D}$ is $30 \mu \mathrm{m}, 25 \mu \mathrm{m}$, and $20 \mu \mathrm{m}$, the corresponding RI sensitivity is $47.3 \mathrm{~nm} / \mathrm{RIU}, 67.1 \mathrm{~nm} / \mathrm{RIU}$, and $96.5 \mathrm{~nm} / \mathrm{RIU}$, respectively. The RI measuring sensitivity reduces linearly with the increase of $D$. Figure 8 is the sensor sensitivity with taper waist diameter of $25 \mu \mathrm{m}$, taper distance of $4.0 \mathrm{~cm}-6.0 \mathrm{~cm}$, taper area length of $818 \mu \mathrm{m}$. When taper distance $\mathrm{L}$ is $4 \mathrm{~cm}, 5 \mathrm{~cm}$ and $6 \mathrm{~cm}$, the corresponding RI sensitivity is $44.3 \mathrm{~nm} / \mathrm{RIU}, 67.1 \mathrm{~nm} / \mathrm{RIU}$, and $81.5 \mathrm{~nm} / \mathrm{RIU}$, respectively. Considering the encapsulation, mechanical strength, transmission loss, $\mathrm{L}$ is bigger and more conducive to sensing. 

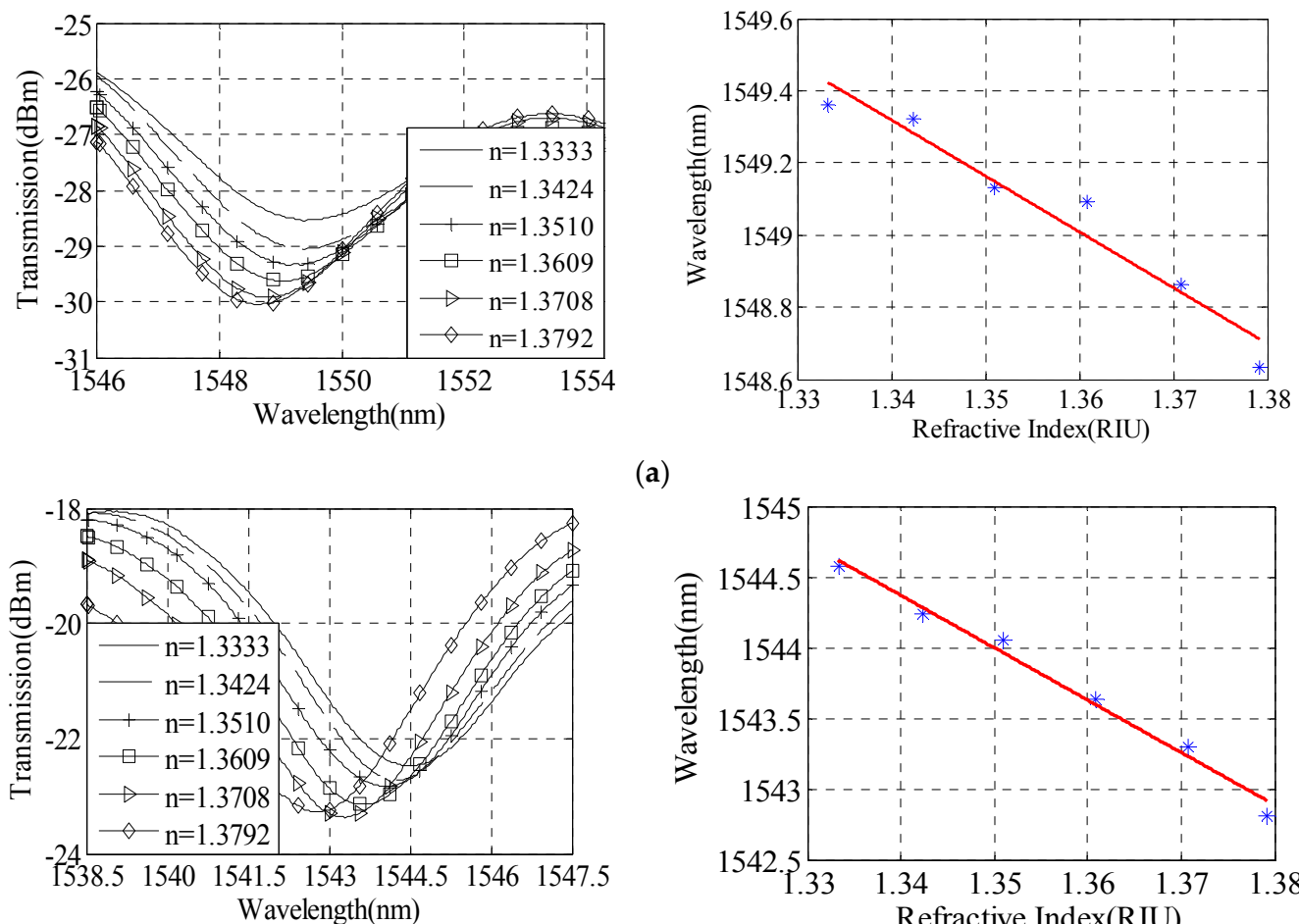

(a)

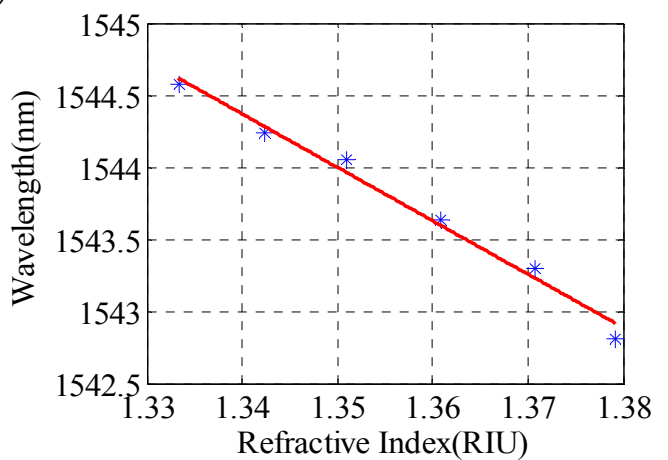

(b)
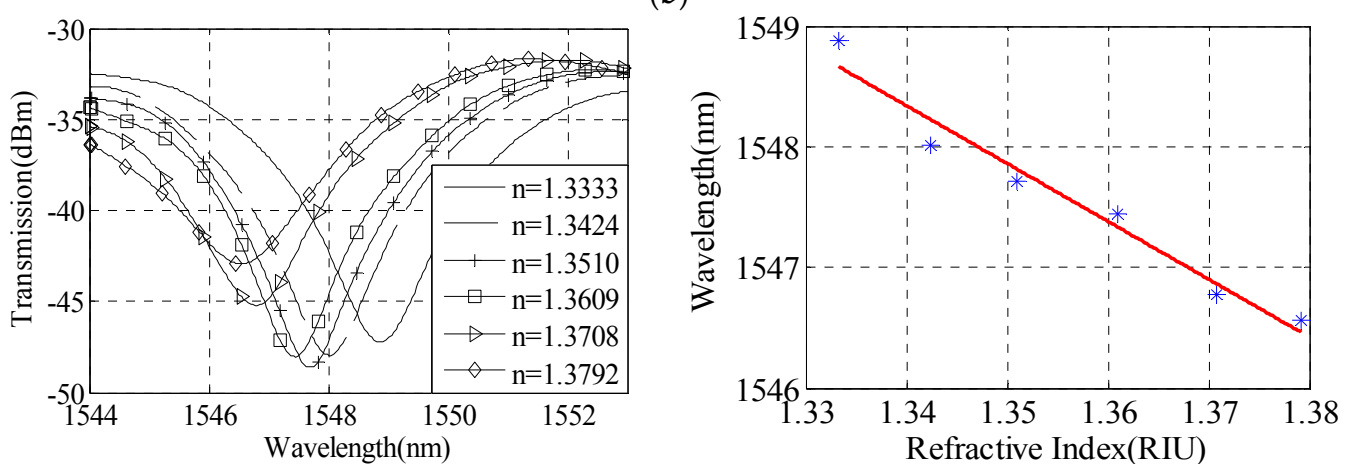

(c)

Figure 7. Refractive index measurement sensitivity analysis with different taper waist diameters. (a) $\mathrm{D}=30 \mu \mathrm{m}$; (b) $\mathrm{D}=25 \mu \mathrm{m}$; (c) $\mathrm{D}=20 \mu \mathrm{m}$.
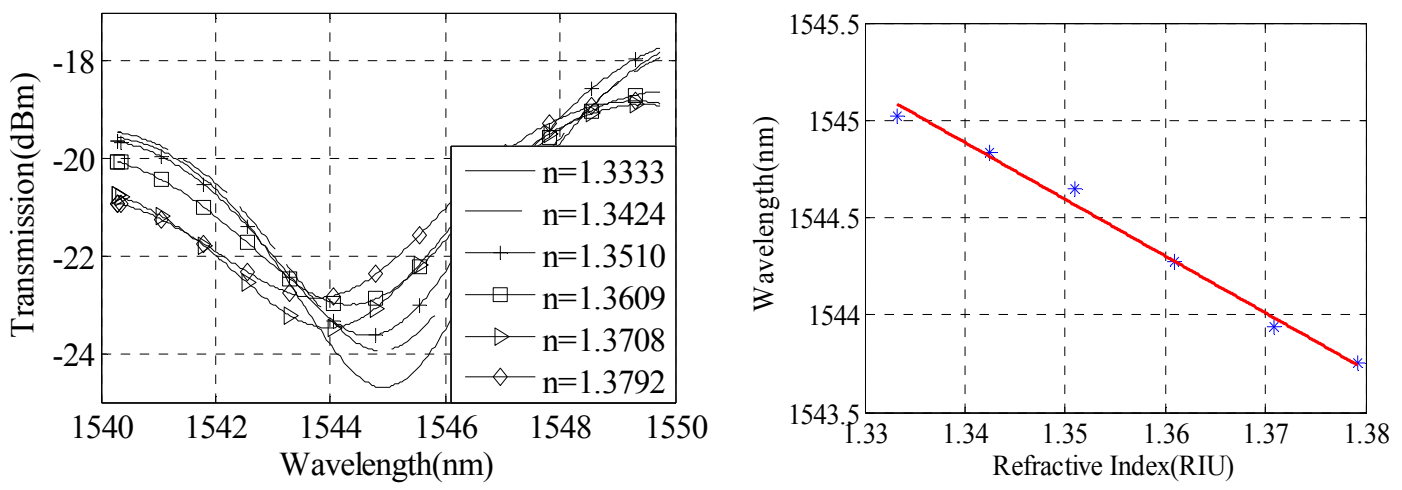

(a)

Figure 8. Cont. 

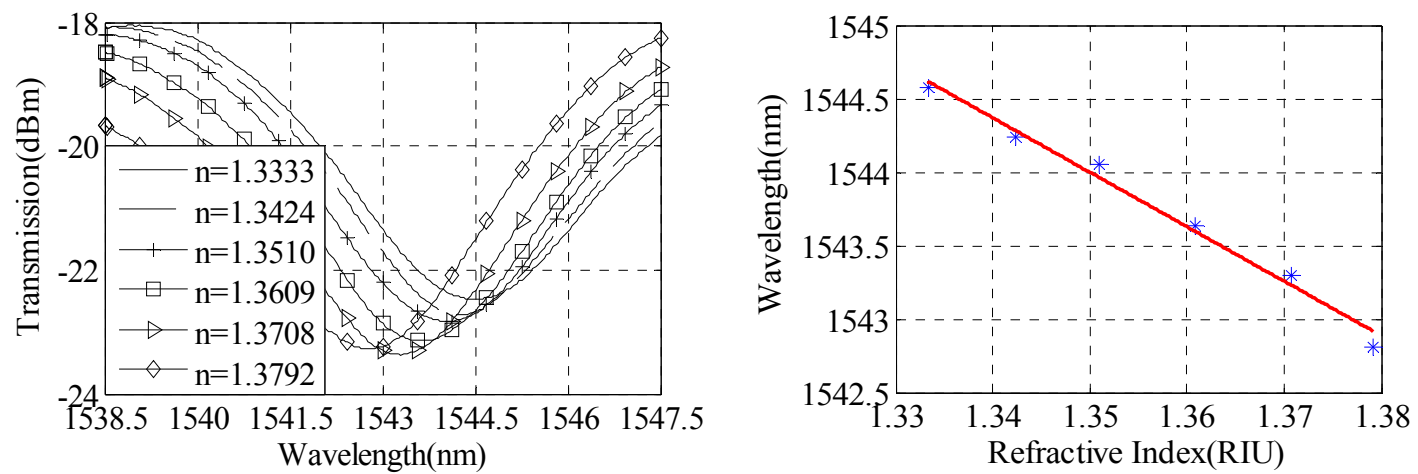

(b)
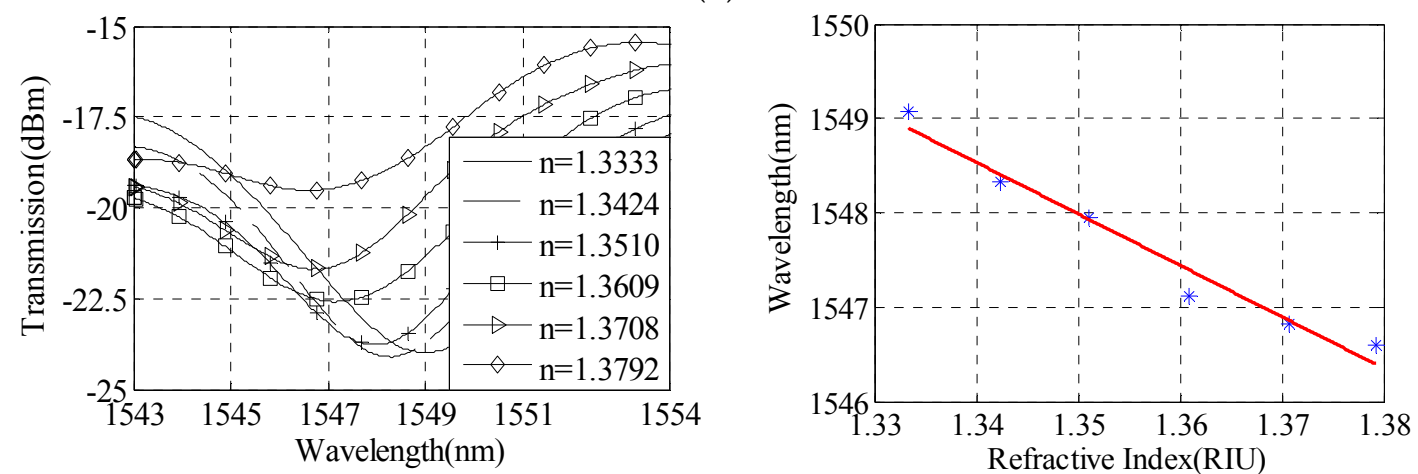

(c)

Figure 8. Refractive index measurement sensitivity analysis with different tapers distances. (a) $\mathrm{L}=4 \mathrm{~cm}$; (b) $\mathrm{L}=5 \mathrm{~cm} ;(\mathbf{c}) \mathrm{L}=6 \mathrm{~cm}$.

In order to reach the higher refractive index sensitivity, we reduced the taper waist diameter as far as possible. The experimental results show that a stable taper waist diameter of $24 \mu \mathrm{m}$ can be obtained by the fusion splicer. Through the continuous experiments, we finally made a cascade dual-taper interferometer with a taper waist diameter of $24 \mu \mathrm{m}$, taper distance of $5.5 \mathrm{~cm}$, and taper area length of $837 \mu \mathrm{m}$. The sensitivity and spectrum characteristics of the RI sensor are shown in Figure 8.

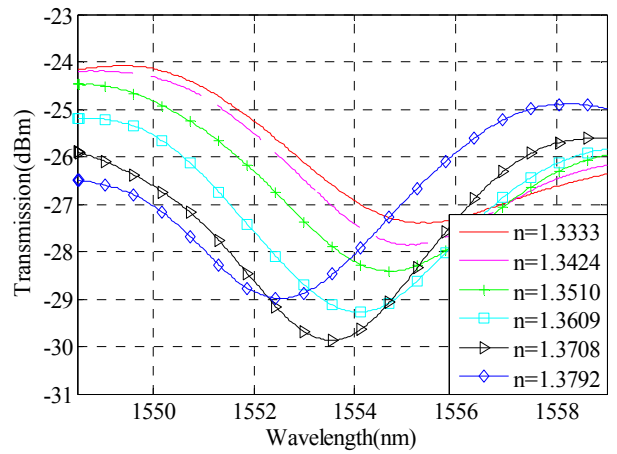

(a)

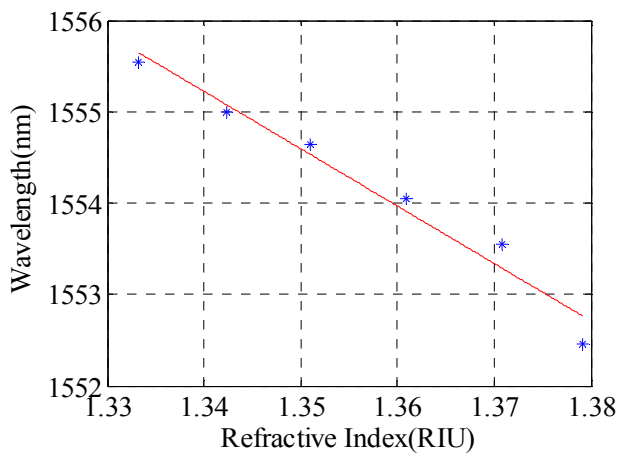

(b)

Figure 9. Sensitivity analysis with taper waist diameter of $24 \mu \mathrm{m}$, taper distance of $5.5 \mathrm{~cm}$, and taper area length of $837 \mu \mathrm{m}$. (a) Interference spectrum; (b) Sensitivity of refractive index measurement.

As shown in Figure 9a, the interference spectrum shows a red shift with the increasing of solution RI, and when the RI increases from 1.3333 to 1.3792, the wavelength of interference dip moves from $1552.455 \mathrm{~nm}$ to $1555.545 \mathrm{~nm}$. The linear relationship is: $y=-62.78 x+1636$. The relationship between 
interference dip and the RI is obtained by Figure $9 \mathrm{~b}$. The RI measurement sensitivity of the sensor can reach $62.78 \mathrm{~nm} / \mathrm{RIU}$.

\section{Conclusions}

In this paper, we proposed a cascaded symmetrical dual-taper Mach-Zehnder interferometer based on interference between the guided-mode and leaky-mode mode. A simple and accurate preparation method of fiber taper was designed, and the influence of taper length, taper distance, and taper waist diameter on the spectrum characteristics and RI measurement sensitivities were simulated. In the experiment, the diameter of the taper is $24 \mu \mathrm{m}$, length of taper area is $837 \mu \mathrm{m}$ and taper distance is $5.5 \mathrm{~cm}$. The experimental results show that the RI measurement sensitivity can reach $62.78 \mathrm{~nm} / \mathrm{RIU}$ in the range of $1.3333 \sim 1.3792$. The resolution can reach $1.6 \times 10^{-5}$ RIU when using an OSA with a resolution of $1 \mathrm{pm}$. Due to the advantages of simple fabrication, low cost, and high sensitivity, the sensor promises to have wide applications in the field of stress, temperature, and so on.

Acknowledgments: This work was supported by the National Natural Science Foundation of China under Grant 61203206, the Fundamental Research Funds for the Central Universities under Grant N140405001, the Specialized Research Fund for the Doctoral Program of Higher Education of China under Grant 20120042120038, the National Science Foundation for Distinguished Young Scholars of China under Grant 61425003, the State Key Laboratory of Synthetical Automation for Process Industries under Grant 2013ZCX09, and the Natural Science Foundation of Hebei Province under Grant F2014501137.

Author Contributions: Qi Wang conceived and designed the experiments; Chunyue Li performed the experiments; Chengwu Zhao analyzed the data; Weizheng Li contributed reagents/materials/analysis tools; Qi Wang wrote the paper.

Conflicts of Interest: The authors declare no conflict of interest.

\section{References}

1. Wei, T.; Lan, X.; Xiao, H. Fiber Inline Core-Cladding-Mode Mach-Zehnder Interferometer Fabricated by Two-Point CO. IEEE Photonics Technol. Lett. 2009, 21, 669-671.

2. Zhou, J.; Wang, Y.; Liao, C.; Zhao, J. Intensity modulated refractive index sensor based on optical fiber Michelson interferometer. Sens. Actuators B Chem. 2015, 208, 315-319. [CrossRef]

3. Li, H.; Yu, X.; Miao, C. Research of intelligent clothing for body temperature monitoring based on distributed optical fiber Bragg grating sensors. Acta Opt. Sin. 2009, 29, 208-212.

4. Li, K. A Study on Miniature Interferometer Strain Sensor Based on EFPI. Acta Opt. Sin. 2009, 29, $3282-3285$.

5. Tian, Z.; Yam, S. In-Line Single-Mode Optical Fiber Interferometric Refractive Index Sensors. J. Lightwave Technol. 2009, 27, 2296-2306. [CrossRef]

6. Tian, Z.; Yam, S.H.; Barnes, J.; Bock, W.; Greig, P.; Fraser, J.M.; Loock, H.; Oleschuk, R.D. Refractive index sensing with mach-zehnder interferometer based on concatenating two single-mode fiber tapers. IEEE Photonics Technol. Lett. 2008, 20, 626-628. [CrossRef]

7. Lu, P.; Men, L.; Sooley, K.; Chen, Q. Tapered fiber M-Z interferometer for simultaneous measurement ofrefractive index and temperature. Appl. Phys. Lett. 2009, 94, 131110-131113. [CrossRef]

8. Wu, D.; Zhu, T.; Deng, M.; Duan, D.; Shi, L.; Yao, J.; Rao, Y. Refractive index sensing based on Mach-Zehnder interferometer embedded in a thinned optical fiber for refractive index sensing. Appl. Opt. 2011, 50, 1548-1553. [CrossRef] [PubMed]

9. Zhang, Q.M.; Jang, S.J.; Cross, L.E. High-frequency strain response in ferroelectrics and its measurement using a modified Machehnder interferometer. J. Appl. Phys. 1989, 65, 2807-2813. [CrossRef]

10. Shin, W.; Lee, Y.L.; Yu, B.A.; Noh, Y.; Ahn, T.J. Highly sensitive strain and bending sensor based on in-line fiber Mach-Zehnder interferometer in solid core large mode area photonic crystal fiber. Opt. Commun. 2010, 283, 2097-2101. [CrossRef]

11. Zhou, Y.W.; Chen, S.Y. A novel In-Line Fiber Mach-Zehnder Interferometer Temperature Sensor Made of Thermally Expanded Core Fiber. Appl. Mech. Mater. 2014, 635, 856-859. [CrossRef]

12. Yadav, T.K.; Narayanaswamy, R.; Bakar, M.H.A.; Kamil, Y.M.; Mahdi, M.A. Single mode tapered fiber-optic interferometer based refractive index sensor and its application to protein sensing. Opt. Express 2014, 22, 22802-22807. [CrossRef] [PubMed] 
13. Yang, J.; Jiang, L.; Wang, S.; Li, B.; Wang, M.; Xiao, H.; Lu, Y.; Tsai, H. High sensitivity of taper-based Mach-Zehnder interferometer embedded in a thinned optical fiber for refractive index sensing. Appl. Opt. 2011, 50, 5503-5507. [CrossRef] [PubMed]

14. Li, B.; Jiang, L.; Wang, S.; Yang, J.; Wang, M.; Chen, Q. High sensitivity Mach-Zehnder interferometer sensors based on concatenated ultra-abrupt tapers on thinned fibers. Opt. Laser Technol. 2012, 44, 640-645. [CrossRef]

15. Li, B.; Jiang, L.; Wang, S.; Wang, Q.C.M.; Yang, J. A new Mach-Zehnder interferometer in a thinned-cladding fiber fabricated by electric arc for high sensitivity refractive index sensing. Opt. Lasers Eng. 2012, 50, 829-832. [CrossRef]

(C) 2016 by the authors; licensee MDPI, Basel, Switzerland. This article is an open access article distributed under the terms and conditions of the Creative Commons Attribution (CC-BY) license (http://creativecommons.org/licenses/by/4.0/). 